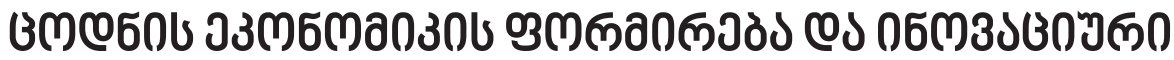

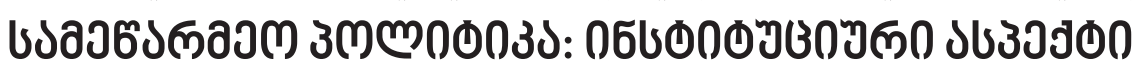

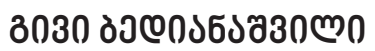

https://doi.org/10.35945/gb.2017.03.001

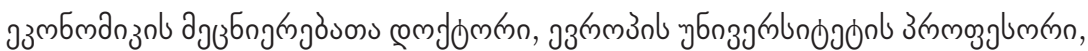

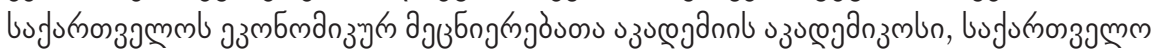

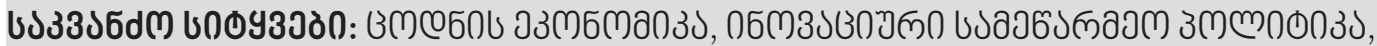

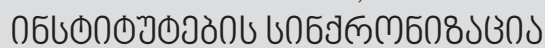

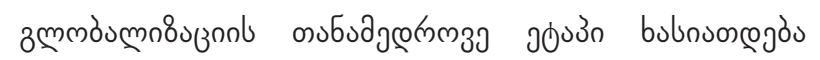

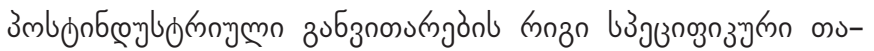

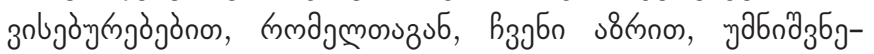

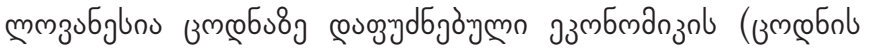

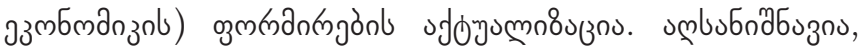

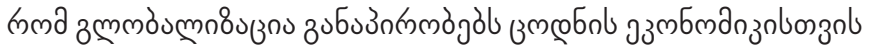

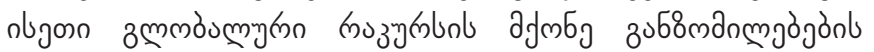

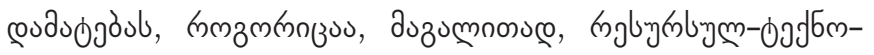

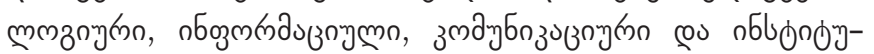

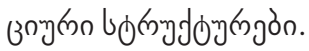

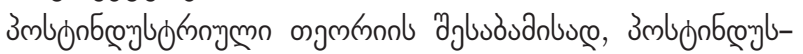

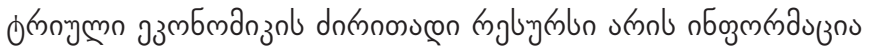

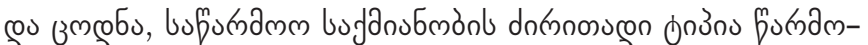

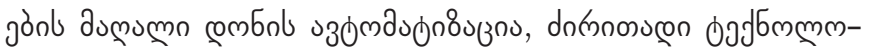

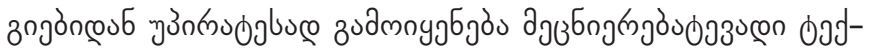

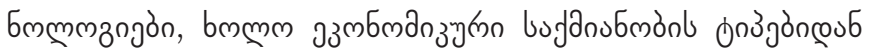

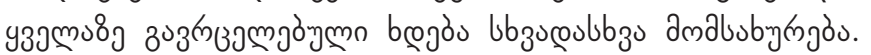

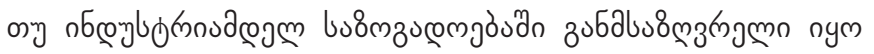

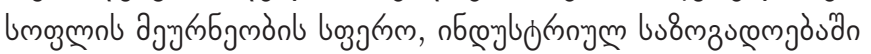

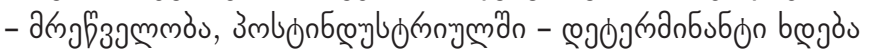

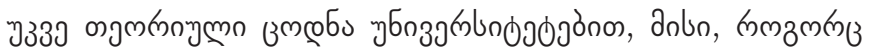

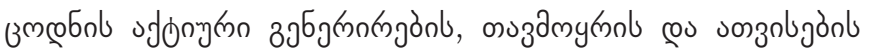
งеgrmmon.

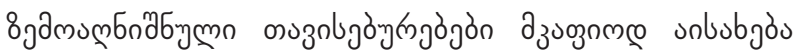

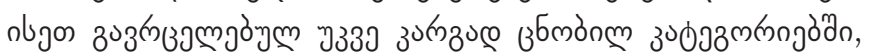

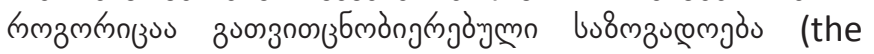

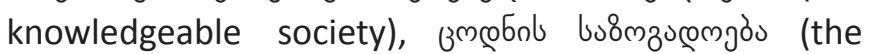

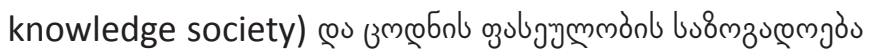

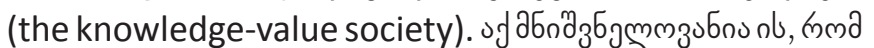

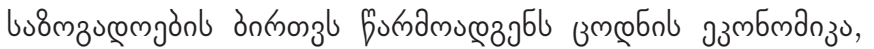

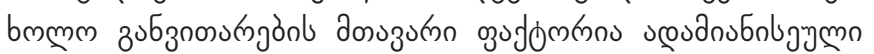
3030000mo.

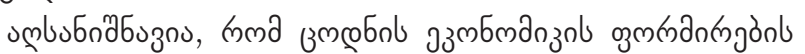

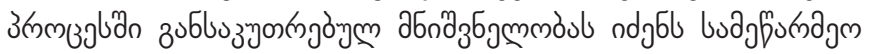
bufanubmòn n n

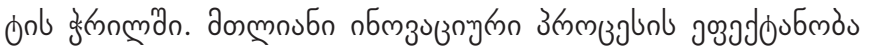

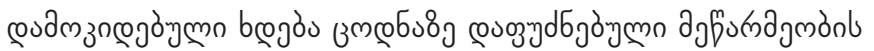

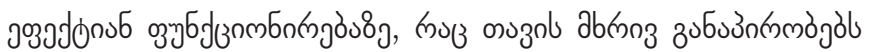

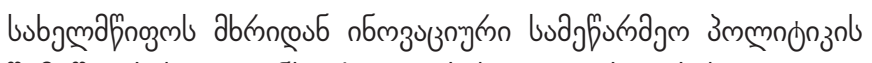

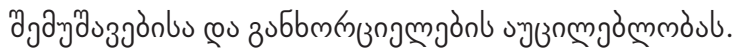

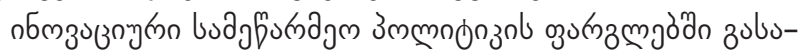

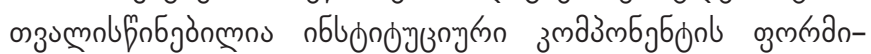

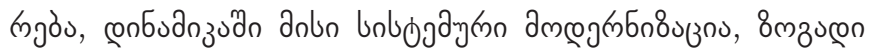

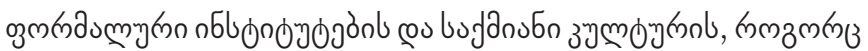

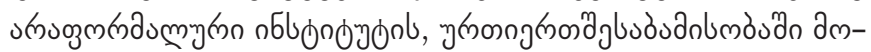

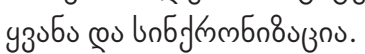

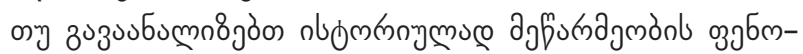

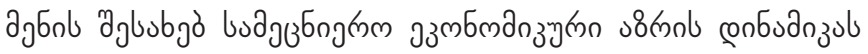
(Baumol, 1990; Блауг, 2008; Веблен, 2007; Друкер, 2007; Саймон, 1995; Макклелланд, 2007; Papava, 2017; Шумпетер, 2007; Audretsch at al., 2001; Dahlstrand at al., 2010; Grilo at al., 2006; Jobnẹn pou bb3., 2009; Светуньков, 2010; Beugelsdijk, 2007; Hofstede,

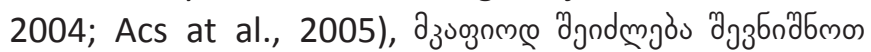

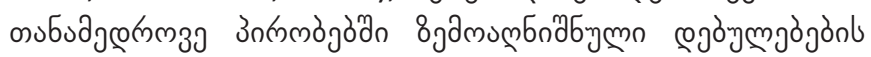

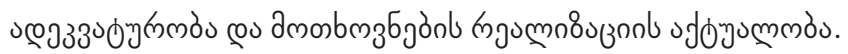

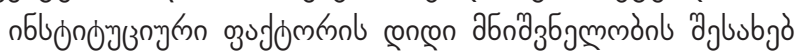

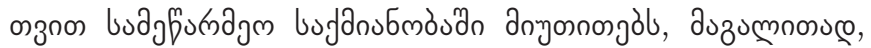

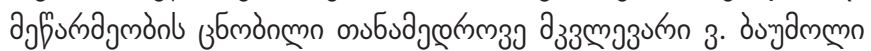

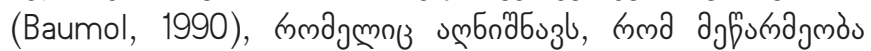

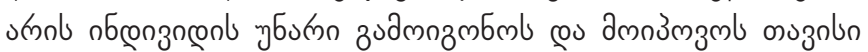

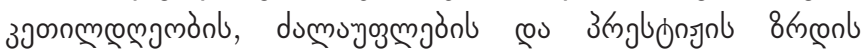

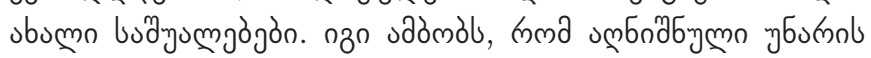

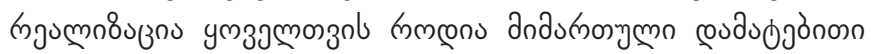

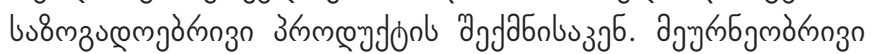

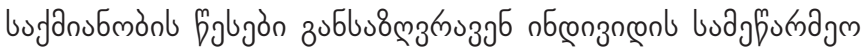

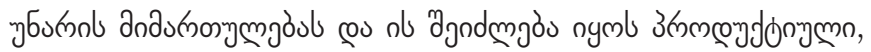

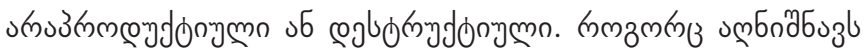

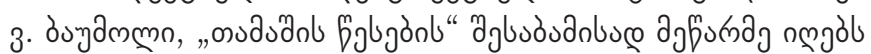

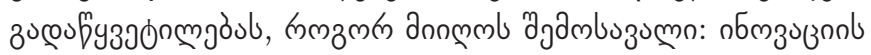

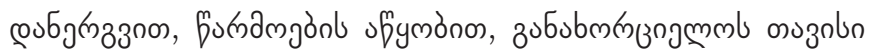

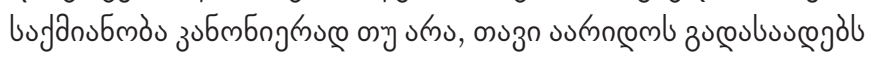

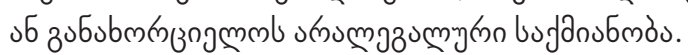

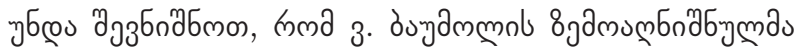

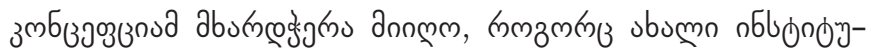

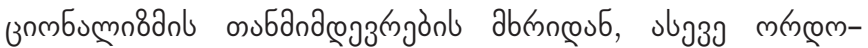




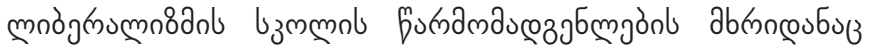

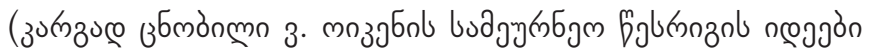

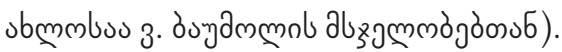

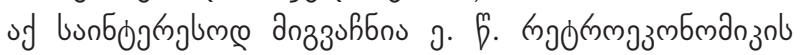

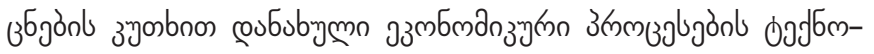

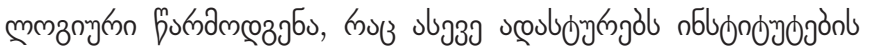

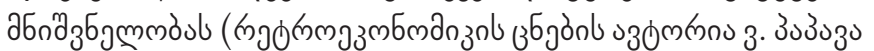

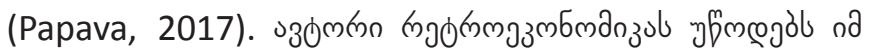

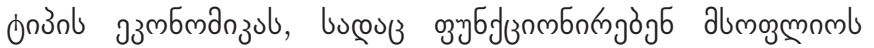

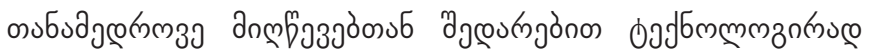

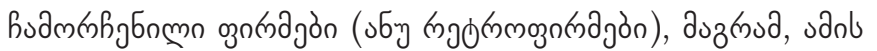

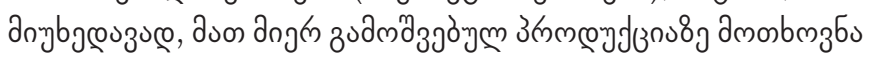
ànб(з unlugàmoll (Papava, 2016: 3).

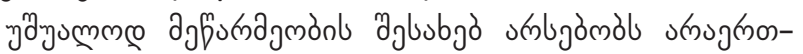

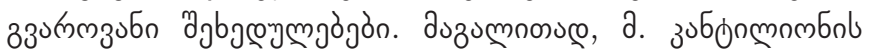

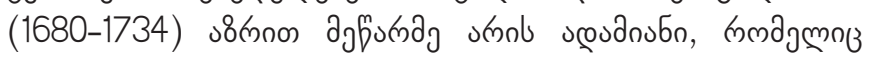

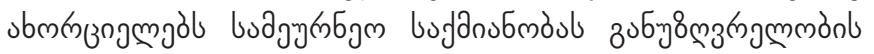
u

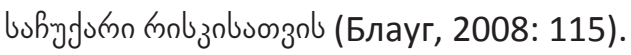

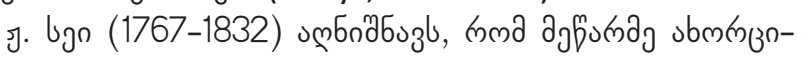

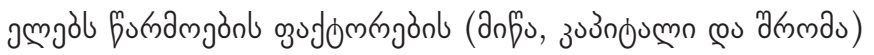

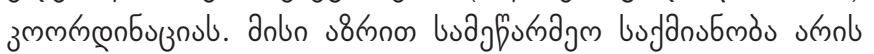

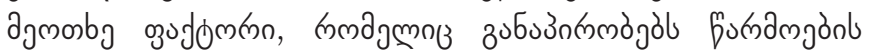

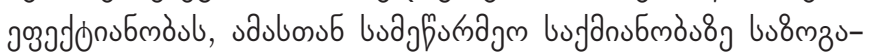

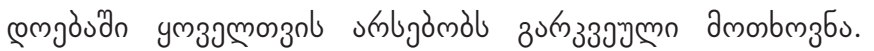

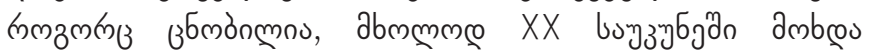

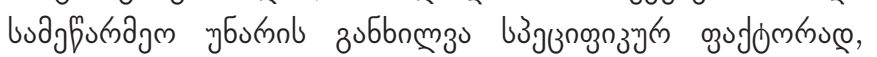

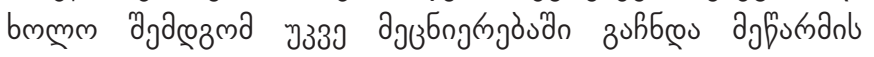

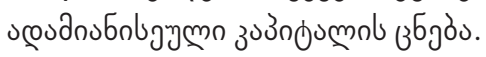

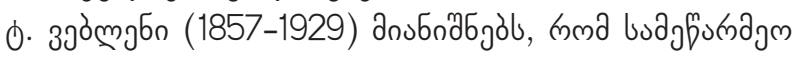

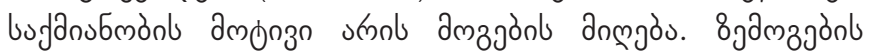

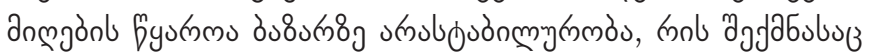

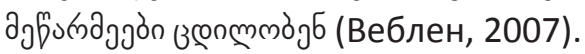

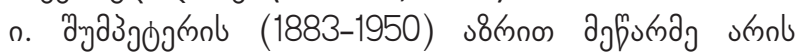

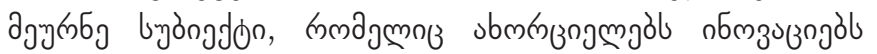

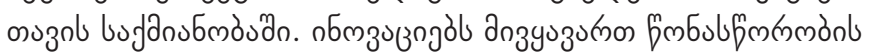

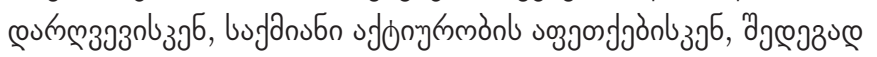

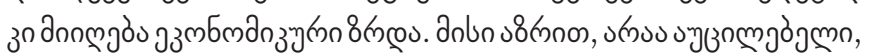

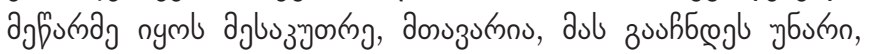

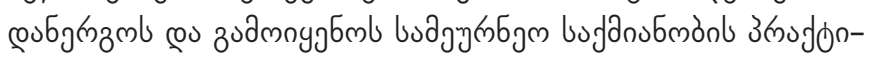

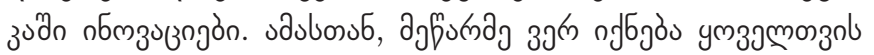

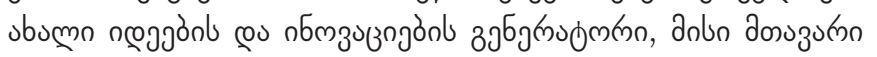

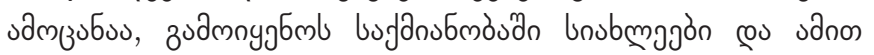

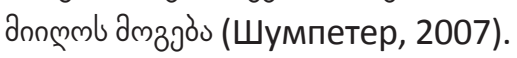

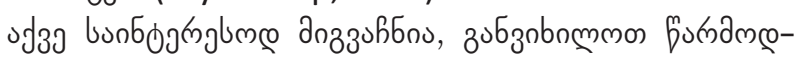

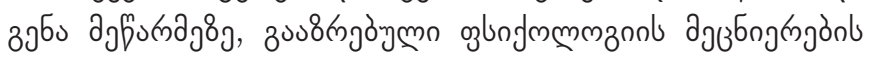
z'yonbnon. duzumnnosue, 3. bundmbn (1916-2001) oुnffrmoll,

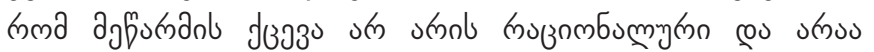

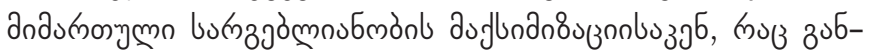

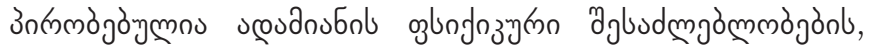

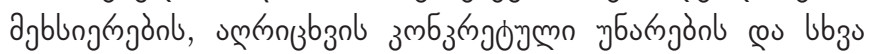

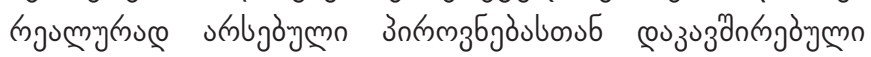

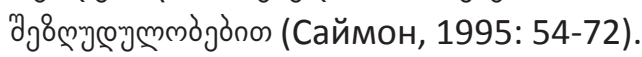

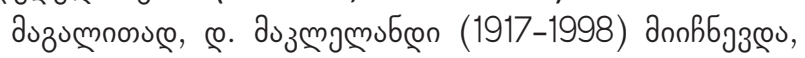

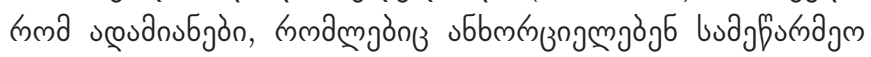

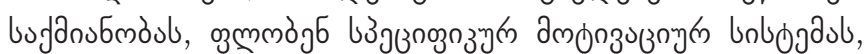

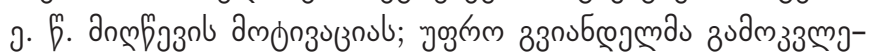

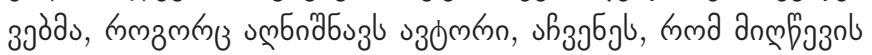

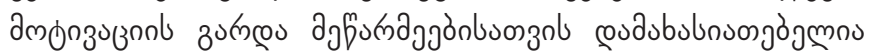

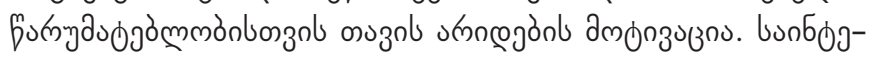

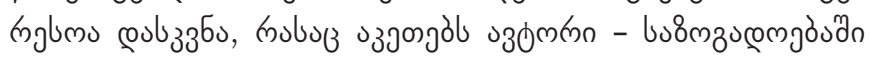

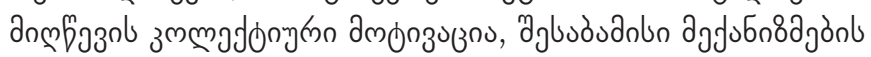

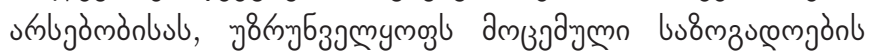

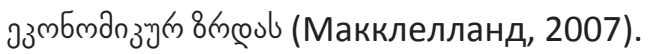

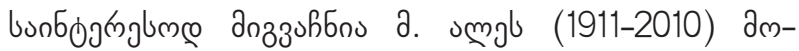

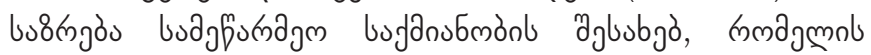

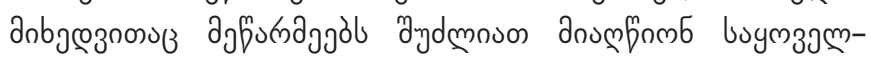

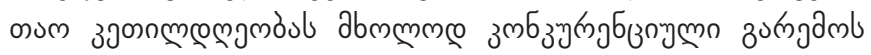
ง

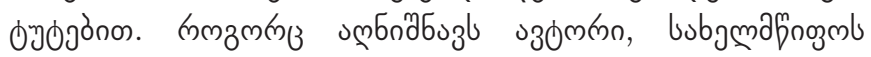

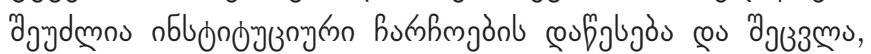
aszकna

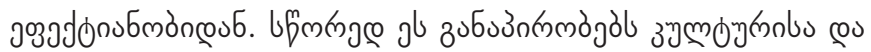

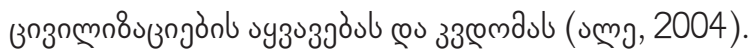

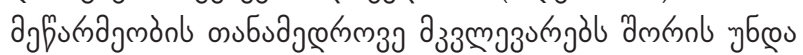
zudmnymb ajб

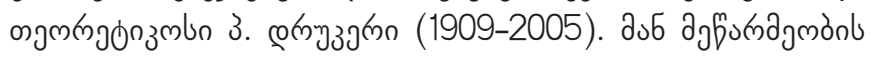

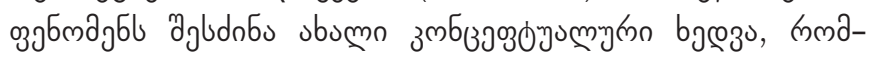

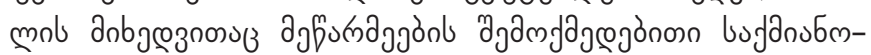

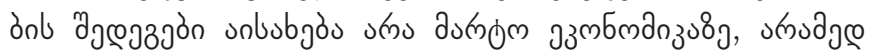

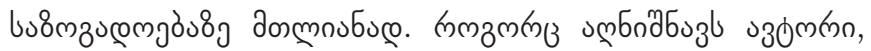

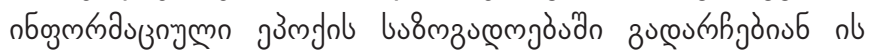

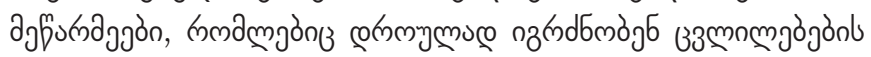

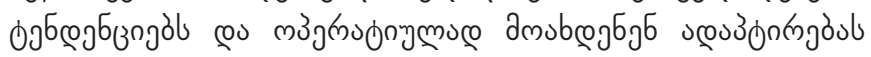

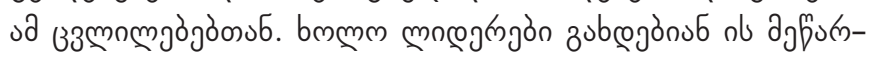

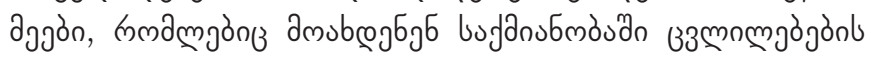
3эб

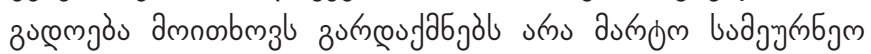

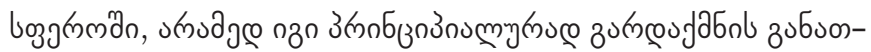

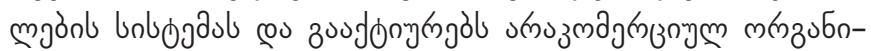

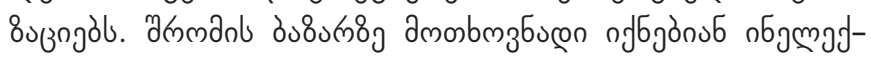

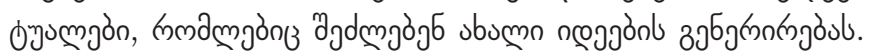

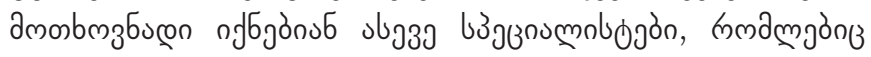

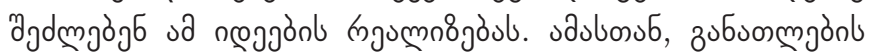

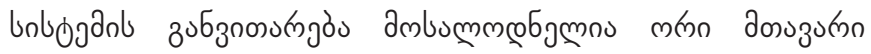

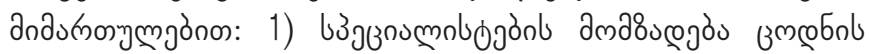

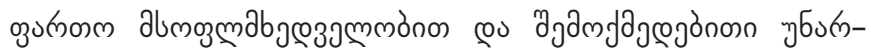

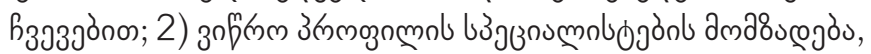




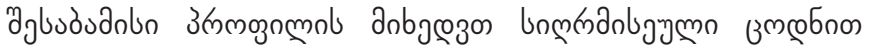
(Друкер, 2007).

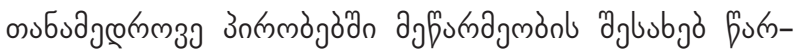

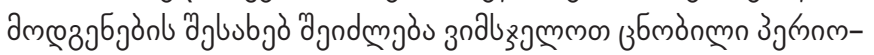

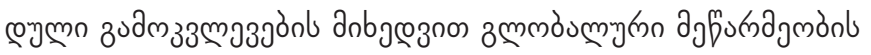

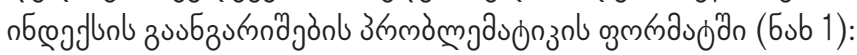

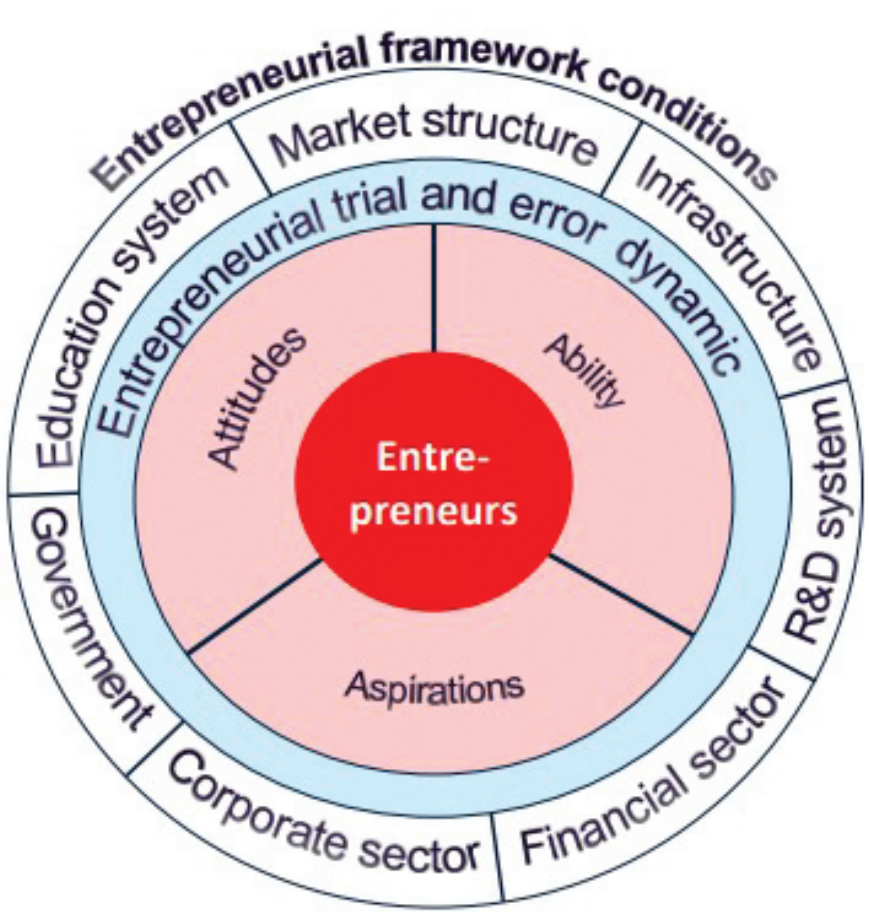

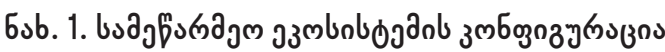

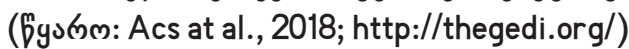

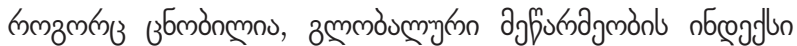

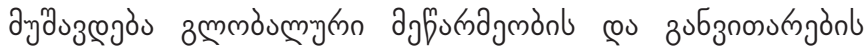
nbbjooyonb angen (The Global Entrepreneurship and Development Institute, Washington, USA). поп зmд3mgd-

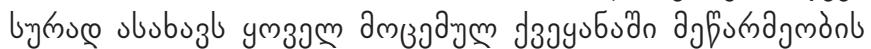

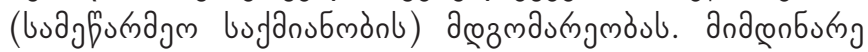
2018 fmnlusonzls ama8uœ

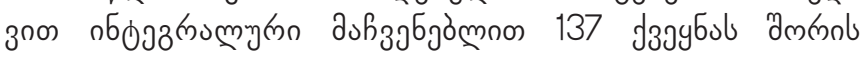

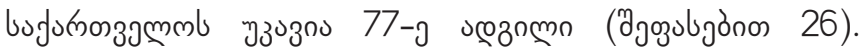

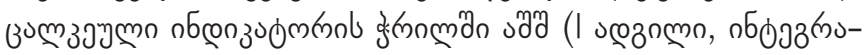

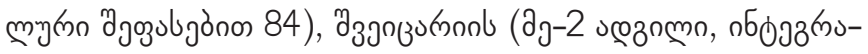

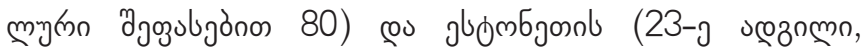

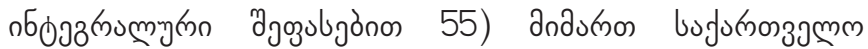

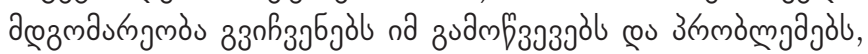

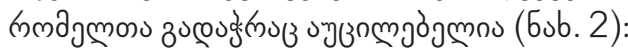

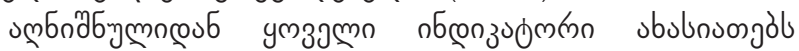

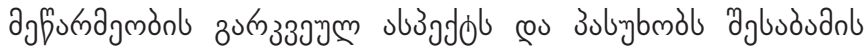

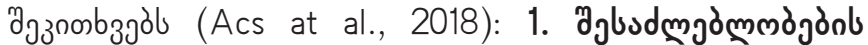

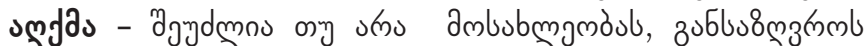

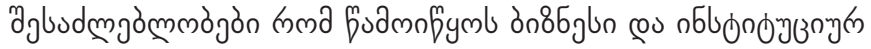

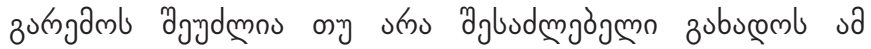

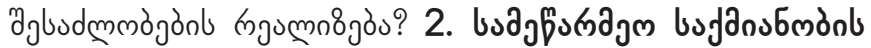

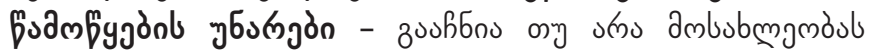

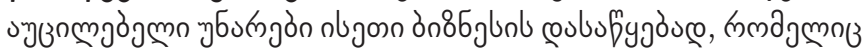

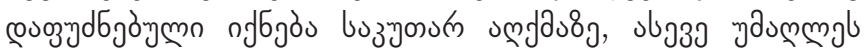

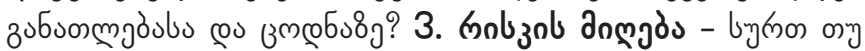

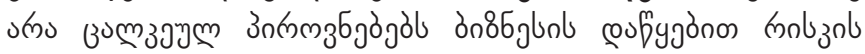

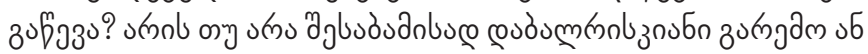

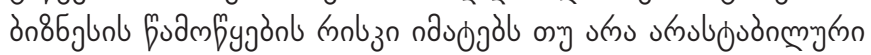

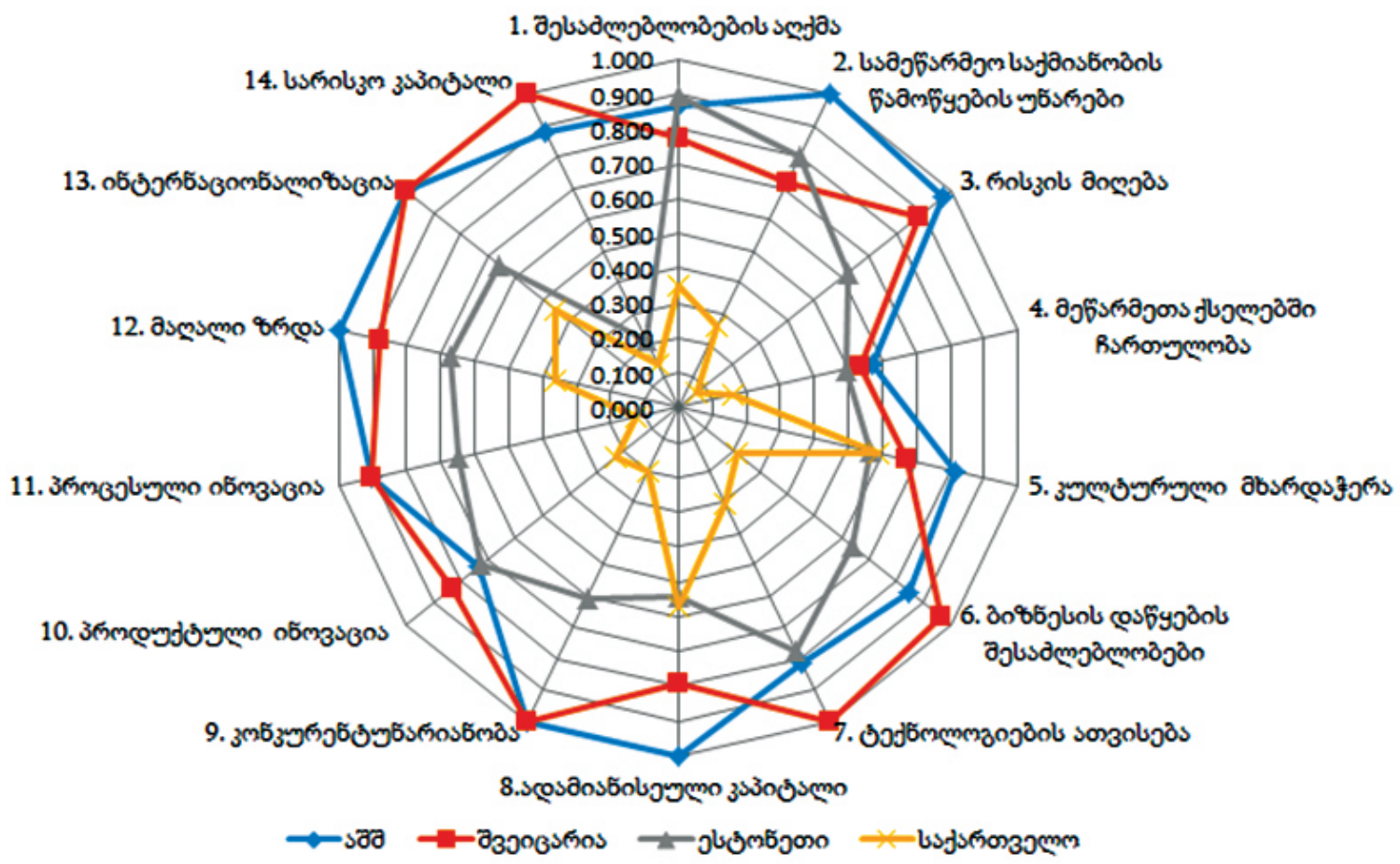

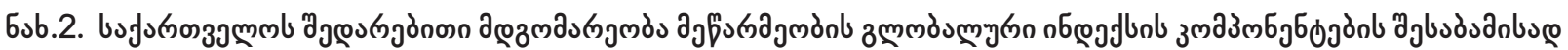

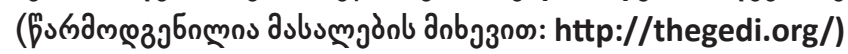




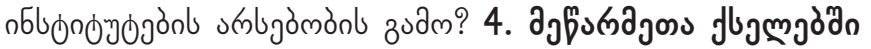

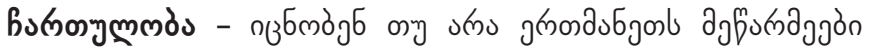

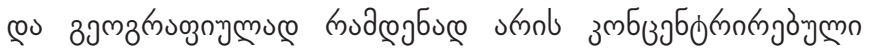

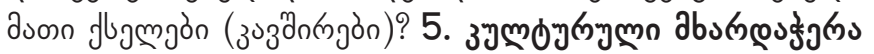

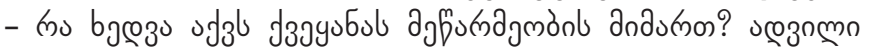
s

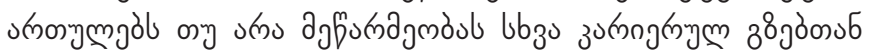

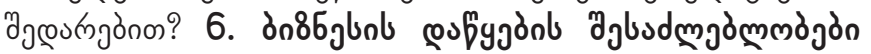

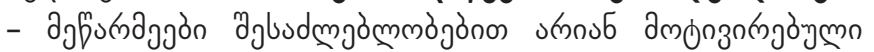

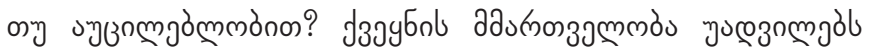

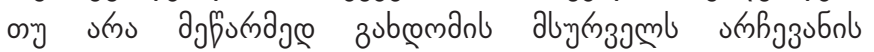

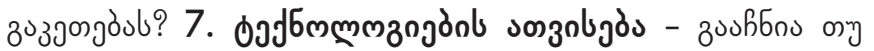

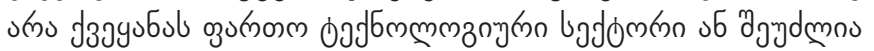

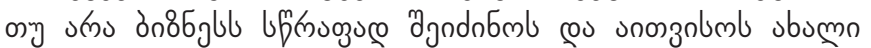

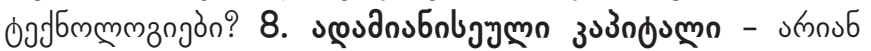

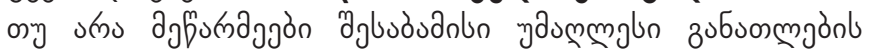

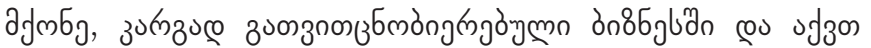

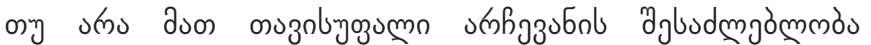

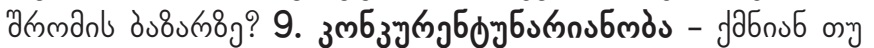

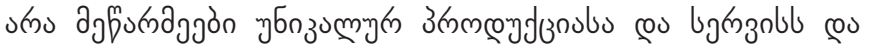

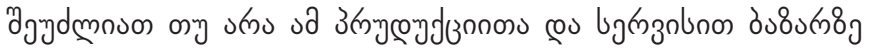

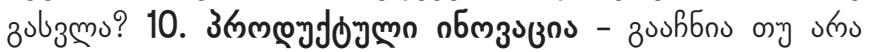

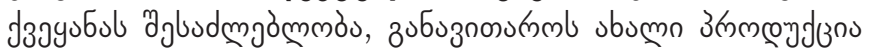

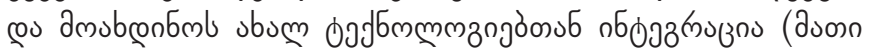

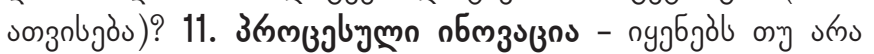

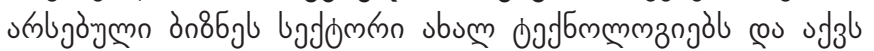

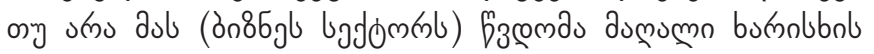

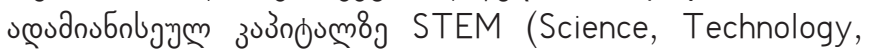

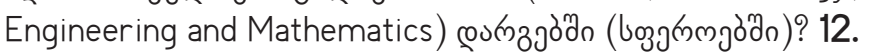

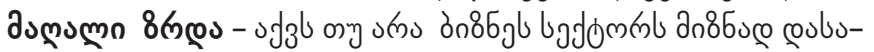

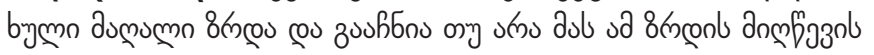

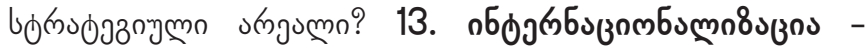

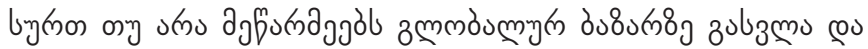

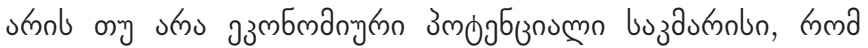

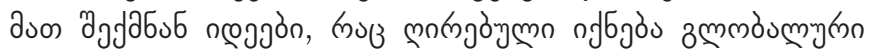

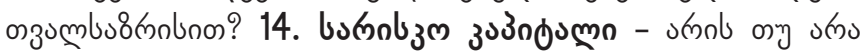

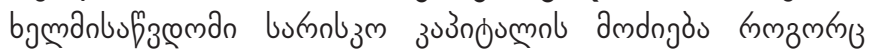

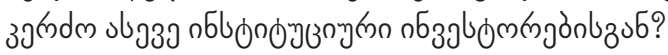

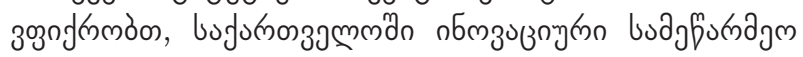

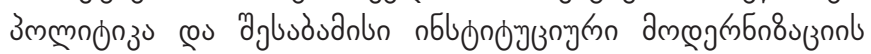

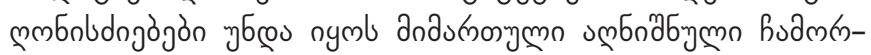

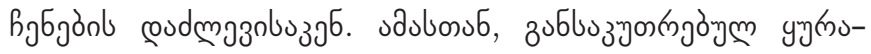

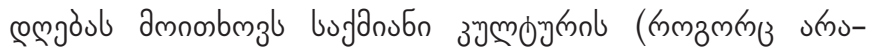

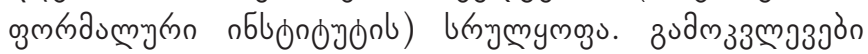
ucoubdyngòj6 (Hofstede, 2004; Bedianashvili, 2014; Didero at al., 2008; Beugelsdijk, 2007; Furman at al., 2002; Geertz, 1973; Herbig and Srholec, 1998; Inglehart and Welzel, 2005; James, 2005; Von Hippel, 2005; Acs, 2006; Barnett, 1953; Beugelsdijk at al., 2014; Brons, 2006; Dickson at al., 2003; Didero at al., 2008; Edler and Fagerberg, 2017; Differences in Inno-

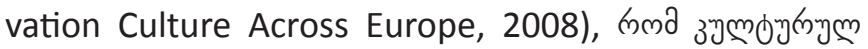

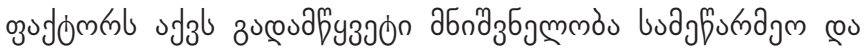

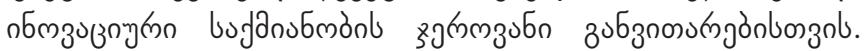

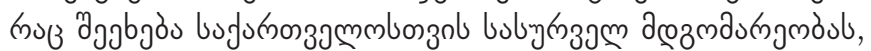

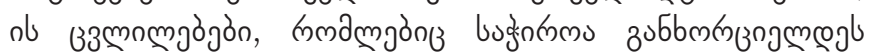

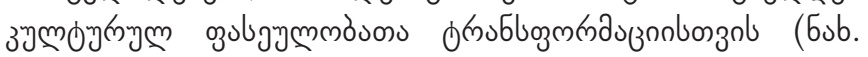

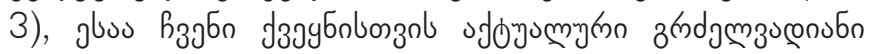

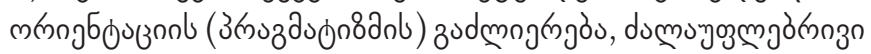

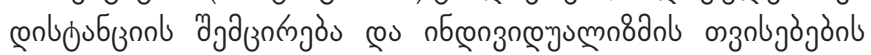
zubznoosungos (Bedianashvili, 2016):

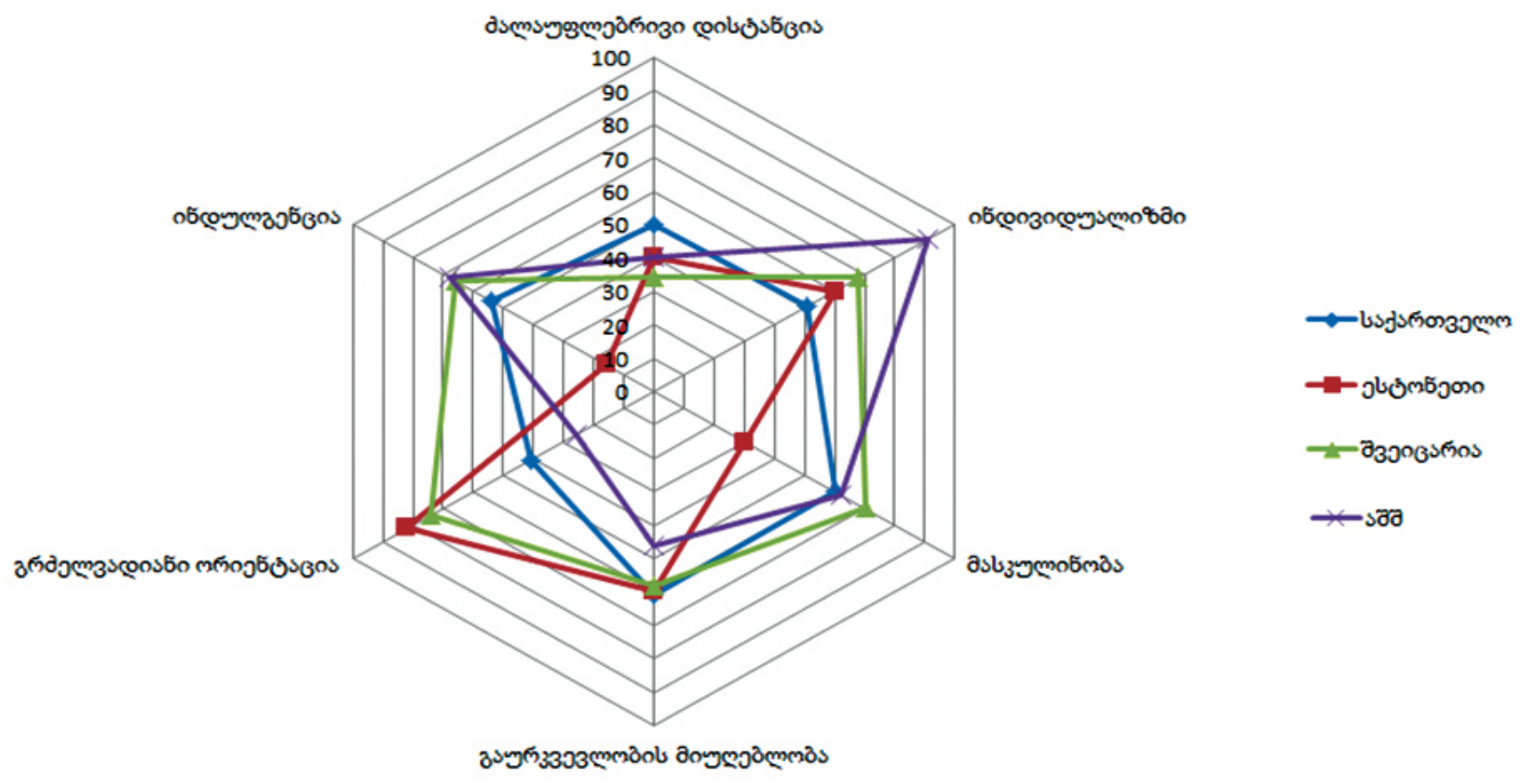

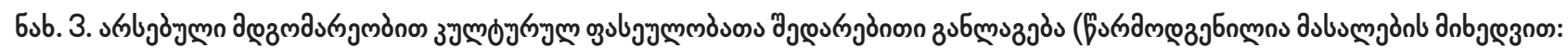

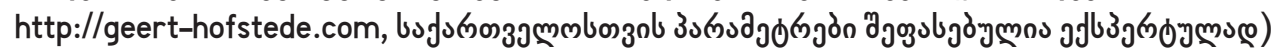




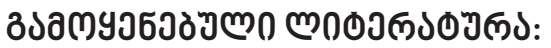

1. Acs, Z. J., Audretsch, D. B., Braunerhjelm, P. and Carlsson, B., (2005). The Knowledge Spillover Theory of Entrepreneurship, Discussion Papers on Entrepreneurship, Growth and Public Policy, Group Entrepreneurship, Growth and Public Policy, Jena, Germany: Max Planck Institute of Economics.

2. Acs, Z. J., Szerb, L., Lloyd, A., (2018). Global Enterpreneurship Index. Washington, GEDI.

3. Baumol W. J. (1990). Enterpreneurship: Productive, Unproductive and Destructive // Journal of Political Economy. Vol. 98. №5. Pt.1. P. 893-920.

4. Bedianashvili, G., (2014). Culture as an Institution in the Context of Socio-economic Development of Country and International Business. J. "Ekonomisti”, No 6. P. 6-16 (Geo).

5. Bedianashvili G. (2016). The Global Business Environment, European Integration and the Cultural Potential of Social-economic Development of Georgia. Journal "Globalization and Business". European University, № 1, P. 19-25 (Geo)

6. Didero, M., Gareis, K., Marques, P., Ratzke, M. et al. (2008). Differences in Innovation Culture across Europe, Discussion paper, TRANSFORM project report, URL: http://www.transformeu.org/publications/documents/Differences in Innovation Culture.pdf (retrieved 10. 7. 2008).

7. Алле М., (2004). Основные направления моей работы / Мировая экономическая мысль. Сквозь призму веков. В 5 т. М.: Мысль.

8. Блауг М. (2008). 100 великих экономистов после Кейнса. - СПб.: Экономическая школа. - 384 с.

9. Веблен Т. (2007). Теория делового предприятия. М.: Дело. -288 с.

10. Друкер П. (2007). Бизнес и инновации. М.: «Вильямс». - 432 с.

11. Саймон Г. (1995). Теория принятия решений в экономической теории и науке о поведении. СПб.: Экономика -534 с.

12. Макклелланд Д. (2007). Мотивация человека. СПб.: Питер. - 672 с.

13. Шумпетер Й. А. (2007). Теория экономического развития. Капитализм, социализм и демократия. М.: Эксмо. $-864 \mathrm{c}$.

14. Светуньков, М., (2010). Предпринимательство и инновации. Ульяновск.: УлГтУ. - 128 с.

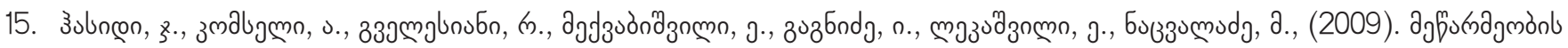

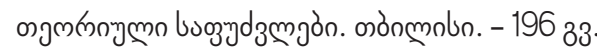

16. European Innovation Scoreboard 2008. Comparative Analysis of Innovation Performance (2009), Pro Inno Europe paper No 10, http://wwwproinno-europe.eu/publications (retrieved, June 2010).

17. Beugelsdijk, S. (2007). 'Entrepreneurial culture, regional innovativeness and economic growth', Journal of Evolutionary Economics, 17(1): 187-210.

18. Furman, J. L., Porter, M. E., Stern, S. (2002), The Determinants of National Innovative Capacity. Research Policy, 31 (6): 899-933. doi:10.1016/ S0048-7333 (01)00152-4

19. Geertz, C. (1973). The Interpretation of Cultures, New York, Basic Books.

20. Hofstede, G et al. (2004) 'Culture's role in entrepreneurship: self-employment out of dissatisfaction', in: Brown, T and Ulijn, J. (eds.) 'Innovation, Entrepreneurship and Culture. The interaction between Technology, Progress and Economic Growth', Cheltenham, Massachusetts: Edward Elgar Publishing, pp. 162-203.

21. Hofstede, G. (2011). Dimensionalizing Cultures: The Hofstede Model in Context. Online Readings in Psychology and Culture, 2(1). https://doi.org/10.9707/2307-0919.1014.

22. Inglehart, R. and Welzel, C. (2005) 'Modernization, Cultural Change, and Democracy: The Human Development Sequence', Cambridge, MA, et al.: Cambridge University Press.

23. Von Hippel, E. (2005) 'Democratizing innovation, Cambridge, MA: MIT Press.

24. Papava V., (2016). Technological Backwardness - Global Reality and Expected Challenges for the World's Economy. No. 70. Tbilisi, GFSIS, 2016 (Geo).

25. Papava V., (2017). Retroeconomics - a Theory of the Technological Backwardness of Economy. Nowe ścieżki rozwoju w Europie Środkowej i Nowe ścieżki rozwoju At Nałęczów, Poland, 2017. - p.33-37.

26. Acs, Z.J., (2006). How is Entrepreneurship Good for Economic Growth?, Innovations: Technology, Governance, Globalization 1(1), p. 97-107. 
27. Dahlstrand, Å. L. and Stevenson, L., (2010). Innovative Entrepreneurship Policy: Linking Innovation and Entrepreneurship in a European Context. Annals of Innovation \& Entrepreneurship, vol.1. Retrieved 08. 09. 2012, from http://www.innovationandentrepreneurship.net/index.php/aie/article/view/5602/html_34.

28. European Union EU. (2012). The Innovation Union Scoreboard. Research and Innovation Union scoreboard, Retrieved 07. 20. 2012, from http://www.proinno-europe.eu/metrics

29. Fagerberg, J. and Srholec, M., (2008). National Innovation Systems, Capabilities and Economic Development, Research Policy 37/9, p.1417-1435.

30. Fagerberg, J., Feldman, M., Shorlec, M., (2011). Technological Dynamics and Social Capability: Comparing U.S. States and European Nations, CIRCLE Paper no 11/2011, Lund University.

31. Barnett, H.G. (1953). Innovation: The basis of cultural change. New York: Mc Graw Hill.

32. Beugelsdijk, S., Slangen, A., Maseland, R., \& Onrust, M. (2014). The impact of home-host cultural distance on foreign affiliate sales: The moderating role of cultural variation within host countries. Journal of Business Research, $67(8), 1638-1646$.

33. Brons, L. (2006). Indirect measurement of regional culture in the Netherlands, Tijdschrift voor Economische en Sociale Geografie, 97(5): 547-566.

34. Dickson, M. W., Den Hartog, D. N., \& Mitchelson, J. K. (2003). Research on leadership in a cross-cultural context: Making progress, and raising new questions. The leadership quarterly, 14(6), 729-768.

35. Didero, M., Gareis, K., Marques, P., \& Ratzke, M. (2008). Differences in innovation culture across Europe. Transformative Use of ICT in EU Regions. Transform, Germany.

36. Edler J., Fagerberg, J. (2017).-Innovation policy: what, why, and how. Oxford Review of Economic Policy, Volume 33, Number 1, pp. 2-23.

37. Differences in Innovation Culture Across Europe (2008). 


\section{FORMATION OF KNOWLEDGE ECONOMY AND INNOVATIVE ENTREPRENEURIAL POLICY: INSTITUTIONAL ASPECTS}

GIVI BEDIANASHVILI

https://doi.org/10.35945/gb.2017.03.001

Doctor of Economic Sciences, Professor Of European University,

Academician of the Academy of Economic Sciences of Georgia, Georgia

\section{KEYWORDS: KNOWLEDGE ECONOMY, INNOVATIVE ENTREPRENEURIAL POLICY, SYNCHRONIZATION INSTITUTIONS}

\section{SUMMARY}

The modern stage of globalization is characterized by a number of specific peculiarities of post-industry development, among which, in our view, the formation of knowledge-based economy (knowledge economy) is important. It is noteworthy that globalization complements the knowledge economy with the dimensions of global scale such as resource-technological, informational, communicative and institutional structures.

According to postindustrial theory, the key resource of post-industrial economy is information and knowledge, and the main type of manufacturing activities is the high level of automated production. Among the main technologies, science inclusive technologies are used, and from the types of economic activities, a range of industry services is most common. If in the pre-industrial society agricultural sector was a determining factor, in the industrial society, it is the industry. In the post-industrial society theoretical knowledge in universities (where knowledge is generated and accumulated) becomes the determinant. These features are clearly reflected in the categories, such as the knowledgeable society, the knowledge society and the knowledge - value society. The important thing here is that the core of the society is the knowledge economy, while human capital is the main factor of development.

It should be noted that in the process of forming the knowledge economy, the innovative aspect of entrepreneurial activities is of a particular importance in the context of all business entities. Therefore, the effectiveness of the overall innovative process depends on the efficient functioning of knowledge-based entrepreneurship, which in its own turn contributes to the development and implementation of innovative entrepreneurial policies by the state. The innovative entrepreneurial policy should envisage the formation of the institutional component, its systematic modernization in dynamics, compliance and synchronization of general formal institutions and business culture as an informal institution. 